\title{
SCHATTEN IDEAL BEHAVIOR OF A GENERALIZED HARDY OPERATOR
}

\author{
KRZYSZTOF NOWAK \\ (Communicated by Palle E. T. Jorgensen)
}

\begin{abstract}
We show the $S^{p}$-criteria and the cut-off result for an operator closely related to the classical Hardy inequality.
\end{abstract}

\section{INTRODUCTION}

For a function $g$ defined on $(0, \infty)$ we consider the operator $A_{g}$ acting on $L^{2}(0, \infty)$, which is given by the formula

$$
A_{g} h(y)=\frac{g(y)}{y} \int_{0}^{y} h(x) d x .
$$

We prove the $S^{p}$-criteria for $A_{g}, 1<p \leq \infty$, and the cut-off result at $p=1$ which states that $A_{g}$ cannot be trace class. This cut-off for $A_{g}$ is related to the result of Rochberg in [R2]. In a more general sense it resembles the critical index behavior of commutators of singular integral operators found by Janson and Wolff in [JW] and the behavior of Hankel operators on Bergman spaces studied by Arazy, Fisher, and Peetre in [AFP]. The case $p=\infty$ is also contained in the paper [BK] by Bloom and Kerman. After this work was done the author was informed that closely related results had been obtained earlier by Birman and Solomyak in [BS].

The boundedness of $A_{g}$ on $L^{2}(0, \infty)$ for $g \equiv 1$ becomes the classical Hardy inequality. The operator $A_{g}$ is essentially a square root of a Calderón-Toeplitz operator $T_{\mu}$ defined in the context of the Haar wavelet, i.e.,

$$
A_{g}^{*} A_{g}=P T_{\mu} P,
$$

where $P: L^{2}(R) \rightarrow L^{2}(0, \infty)$ is the orthogonal projection, and also $T_{\mu}$ may be expressed in terms of $A_{g}^{*} A_{g}$. The symbol of $T_{\mu}$ is the measure $\mu$ defined on the upper half plane by the formula

$$
\int_{-\infty}^{\infty} \int_{0}^{\infty} F(u, s) d \mu(u, s)=\int_{0}^{\infty} F(0, s)|g(s)|^{2} \frac{d s}{s} .
$$

Received by the editors September 26, 1991.

1991 Mathematics Subject Classification. Primary 47B10, 47B35.

Key words and phrases. Schatten ideal, Hardy inequality, Haar wavelet. 
The background for Calderón-Toeplitz operators is presented in [R1, N]. The result in $[\mathrm{N}]$ for Calderón-Toeplitz operators shows that for a positive measure $\mu$ defined on the upper half plane and $p \geq 1$

$$
T_{\mu} \in S^{p} \quad \text { if and only if } \int_{0}^{\infty} \int_{-\infty}^{\infty}|\mu(D(u, s))|^{p} d u \frac{d s}{s^{2}}<\infty,
$$

where $D(u, s)$ is a hyperbolic disk with fixed radius centered at $(u, s)$.

\section{Preliminaries}

For a compact operator $T$ acting on a Hilbert space the $n$th singular value of $T$ is defined as

$$
s_{n}(T)=\inf \{\|T-S\|: \operatorname{rank}(S) \leq n\} .
$$

The Schatten ideal $S^{p}, 1 \leq p<\infty$, is defined as the set of those compact operators $T$ such that

$$
\|T\|_{S^{p}}=\left(\sum_{n}\left(s_{n}(T)\right)^{p}\right)^{1 / p}<\infty .
$$

In addition $S^{\infty}$ stands for the algebra of bounded operators. We refer to [GK, $\mathrm{Mc}, \mathrm{Si}, \mathrm{Z}]$ for more information about Schatten ideals.

For $g$ a measurable function defined on $(0, \infty)$

$$
\|g\|_{l^{p}\left(L^{2}\right)}=\left(\sum_{k}\left(\int_{2^{k}}^{2^{k+1}}|g(x)|^{2} \frac{d x}{x}\right)^{p / 2}\right)^{1 / p},
$$

and $l^{p}\left(L^{2}\right)$ consists of those $g$ for which $\|g\|_{l^{p}\left(L^{2}\right)}$ is finite.

A small letter $c$ denotes a constant which changes its value from place to place. A symbol $\chi_{A}$ stands for the characteristic function of a set $A$.

Proposition 1.1 (see [Mc]). Let $0<p \leq 1$ and let $T$ be a compact positive operator on a Hilbert space $H$. If for some orthonormal basis $\left\{\psi_{i}\right\}$ of $H$

$$
\sum_{i}\left|\left\langle T \psi_{i}, \psi_{i}\right\rangle\right|^{p}<\infty
$$

then $T \in S^{p}$ and

$$
\|T\|_{S^{p}}^{p} \leq \sum_{i}\left|\left\langle T \psi_{i}, \psi_{i}\right\rangle\right|^{p} .
$$

Proposition 1.2. Let $1 \leq p \leq \infty$ and let $\left\{f_{k}\right\},\left\{g_{k}\right\}$ be orthonormal sets in a Hilbert space $H$. If $T \in S^{p}$, then

$$
\left(\sum_{k}\left|\left\langle T f_{k}, g_{k}\right\rangle\right|^{p}\right)^{1 / p} \leq\|T\|_{S^{p}}
$$

If $T$ is compact, then

$$
\lim _{|k| \rightarrow \infty}\left\langle T f_{k}, g_{k}\right\rangle=0
$$

Proof. The first part follows by a standard duality argument, and the second by an approximation argument. 
Proposition 1.3. Let $1 \leq p_{0}, p_{1} \leq \infty, 0 \leq \theta \leq 1, \frac{1}{p}=\frac{1-\theta}{p_{0}}+\frac{\theta}{p_{1}}$. If $T$ is $a$ bounded operator from $l^{p_{0}}\left(L^{2}\right)$ into $S^{p_{0}}$ and from $l^{p_{1}}\left(L^{2}\right)$ into $S^{p_{1}}$, then it is also bounded from $l^{p}\left(L^{2}\right)$ into $S^{p}$ and

$$
\|T\|_{l^{p}\left(L^{2}\right) \rightarrow S^{p}} \leq\|T\|_{l^{p_{0}}\left(L^{2}\right) \rightarrow S^{p_{0}}}^{1-\theta}\|T\|_{l^{p_{1}\left(L^{2}\right) \rightarrow S^{p_{1}}}}^{\theta} .
$$

Proof. The proof follows from standard interpolation results; in particular, Theorem 5.1.2 in [BL] and Theorem 4.2.7 in [Z].

\section{THE MAIN RESULT}

Theorem 2.1. Let $1<p \leq \infty$ and let $g$ be a locally square integrable function.

(1) The operator $A_{g}$ is in the Schatten ideal $S^{p}$ if and only if $g$ belongs to $l^{p}\left(L^{2}\right) ;$ moreover, the corresponding norms are equivalent. The operator $A_{g}$ is compact if and only if

$$
\lim _{|k| \rightarrow \infty} \int_{2^{k}}^{2^{k+1}}|g(y)|^{2} \frac{d y}{y}=0 .
$$

(2) If $A_{g} \in S^{1}$, then $g \equiv 0$.

Condition (2) states that the family of operators $\left\{A_{g}\right\}$ is "cut off" at $p=1$.

Proof. We prove that the map $A, A(g)=A_{g}$, is bounded from $l^{\infty}\left(L^{2}\right)$ into $S^{\infty}$ and from $l^{p}\left(L^{2}\right)$ into $S^{p}, 1<p \leq 2$. It follows by Proposition 1.3 that it is bounded from $l^{p}\left(L^{2}\right)$ into $S^{p}$ for all $1<p \leq \infty$.

We first consider $p=\infty$. We observe that

$$
\begin{aligned}
\int_{0}^{\infty}\left|\frac{g(y)}{y} \int_{0}^{y} h(x) d x\right|^{2} d y & =\sum_{k} \int_{2^{k}}^{2^{k+1}} \frac{|g(y)|^{2}}{y^{2}}\left(\int_{0}^{y}|h(x)| d x\right)^{2} \\
& \leq \sup _{k} \int_{2^{k}}^{2^{k+1}} \frac{|g(y)|^{2}}{y} d y \sum_{k} \frac{1}{2^{k}}\left(\int_{0}^{2^{k+1}}|h(x)| d x\right)^{2}
\end{aligned}
$$

and that

$$
\begin{aligned}
& \left(\sum_{k} \frac{1}{2^{k}}\left(\int_{0}^{2^{k+1}}|h(x)| d x\right)^{2}\right)^{1 / 2} \\
& \quad \leq c\left(\int_{0}^{\infty}\left(\frac{1}{y} \int_{0}^{y}|h(x)| d x\right)^{2} d y\right)^{1 / 2} \leq c\|h\|,
\end{aligned}
$$

where the last inequality is justified by the classical Hardy inequality. The above computation shows that $A$ is bounded from $l^{\infty}\left(L^{2}\right)$ into $S^{\infty}$.

Now let $1<p \leq 2$. It is clear that $A_{g}^{*} A_{g}$ is given by the kernel

$$
K(x, z)=\int_{0}^{\infty} \frac{|g(y)|^{2}}{y^{2}} \chi_{[0, y]}(x) \chi_{[0, y]}(z) d y .
$$

Let

$$
K_{k}(z, x)=\int_{2^{k}}^{2^{k+1}} \frac{|g(y)|^{2}}{y^{2}} \chi_{[0, y]}(x) \chi_{[0, y]}(z) d y .
$$


The operator given by $K_{k}$ acts on $L^{2}\left(0,2^{k+1}\right)$, and

$$
e_{n}^{k}(x)=2^{-(k+1) / 2} e^{2 \pi i 2^{-(k+1)} n x}
$$

forms an orthonormal basis on that space. A direct computation gives

$$
\left|\left\langle K_{k} e_{n}^{k}, e_{n}^{k}\right\rangle\right| \leq \frac{c}{n^{2}} \int_{2^{k}}^{2^{k+1}}|g(y)|^{2} \frac{d y}{y} .
$$

We obtain by Proposition 1.1

$$
\left\|K_{k}\right\|_{S^{p / 2}}^{p / 2} \leq \sum_{n}\left|\left\langle K_{k} e_{n}^{k}, e_{n}^{k}\right\rangle\right|^{p / 2} \leq c\left(\int_{2^{k}}^{2^{k+1}}|g(y)|^{2} \frac{d y}{y}\right)^{p / 2},
$$

and this provides the estimate

$$
\left\|A_{g}\right\|_{S^{p}}^{p}=\|K\|_{S^{p / 2}}^{p / 2} \leq \sum_{k}\left\|K_{k}\right\|_{S^{p / 2}}^{p / 2} \leq c\|g\|_{l^{p}\left(L^{2}\right)}^{p} .
$$

Thus $A$ is bounded from $l^{p}\left(L^{2}\right)$ into $S^{p}, 1<p \leq 2$.

It is easy to observe at this point that if

$$
\lim _{|k| \rightarrow \infty} \int_{2^{k}}^{2^{k+1}}|g(y)|^{2} \frac{d y}{y}=0,
$$

then $A_{g}$ is compact.

Now for the converse estimate we assume that $A_{g} \in S^{p}$, and we take

$$
\begin{aligned}
& f_{k}(x)=2^{-(k-1) / 2} \chi_{\left[2^{k-1}, 2^{k}\right]}(x), \\
& g_{k}(y)=\frac{g(y)}{y} \chi_{\left[2^{k}, 2^{k+1}\right]}(y)\left(\int_{2^{k}}^{2^{k+1}} \frac{|g(y)|^{2}}{y^{2}} d y\right)^{-1 / 2} .
\end{aligned}
$$

It follows by Proposition 1.2 that

$$
\sum_{k}\left|\left\langle A_{g} f_{k}, g_{k}\right\rangle\right|^{p} \leq\left\|A_{g}\right\|_{S^{p}}^{p} .
$$

A direct computation gives

$$
\left\langle A_{g} f_{k}, g_{k}\right\rangle \cong\left(\int_{2^{k}}^{2^{k+1}}|g(y)|^{2} \frac{d y}{y}\right)^{1 / 2},
$$

and this proves the converse estimate.

Now we discuss the cut-off. Assume that for some $0<a<b<\infty$

$$
0<\int_{a}^{b}|g(y)|^{2} \frac{d y}{y}<\infty
$$

and that $A_{g} \in S^{1}$. The kernel

$$
B_{g}(y, x)=\chi_{(a, b)}(y) \frac{g(y)}{y} \chi_{[0, y]}(x) \chi_{(0, b)}(x)
$$

defines an operator in $S^{1}(0, b)$. Let $e_{n}(x)=e^{2 \pi i b n x}$. It is easy to check that

$$
\left\langle B_{g} e_{n}, e_{n}\right\rangle=c\left(\hat{g}_{0}(0)-\hat{g}_{0}(n)\right) / n,
$$


where $g_{0}(y)=\frac{g(y)}{y} \chi_{(a, b)}(y)$. Changing $a, b$ if necessary we may arrange that

$$
\hat{g}_{0}(0)=\int_{a}^{b} \frac{g(y)}{y} d y \neq 0 .
$$

By the Riemann-Lebesgue lemma $\hat{g}_{0}(n) \rightarrow 0$, so

$$
\sum_{n}\left|\left\langle B_{g} e_{n}, e_{n}\right\rangle\right|=\infty
$$

and this contradicts Proposition 1.2. Thus $A_{g}$ is not in the trace class.

Remark 2.2. The $S^{p}$-criteria for $A_{g}, p \geq 2$, may also be obtained by an application of the results for Calderón-Toeplitz operators in [N].

\section{ACKNOWLEDGMENT}

The author thanks Professor Richard Rochberg for valuable conversations on the subject.

\section{REFERENCES}

[AFP] J. Arazy, S. Fisher, and J. Peetre, Hankel operators on weighted Bergman spaces, Amer. J. Math. 110 (1988), 989-1055.

[BK] S. Bloom and R. Kerman, Weighted norm inequalities for operators of Hardy type, Proc. Amer. Math. Soc. 113 (1991), 135-141.

[BL] J. Bergh and J. Löfström, Interpolation spaces: An introduction, Grundlehren Math. Wiss., vol. 223, Springer-Verlag, Berlin, 1976.

[BS] M. Sh. Birman and M. Z. Solomyak, Estimates for the number of negative eigenvalues of the Schrödinger operator and its generalizations, Adv. Soviet Math. 7 (1991), 1-55.

[FS] J. J. F. Fournier and J. Stewart, Amalgams of $L^{p}$ and $l^{q}$, Bull. Amer. Math. Soc. (N.S.) 13 (1985), 1-21.

[GK] I. Gohberg and M. G. Krein, Introduction to the theory of linear nonselfadjoint operators, Transl. Math. Monographs, vol. 18, Amer. Math. Soc., Providence, RI, 1969.

[JW] S. Janson and T. Wolff, Schatten classes and commutators of singular integral operators, Ark. Mat. 82 (1982), 301-310.

[Mc] C. A. McCarthy, $c_{p}$, Israel J. Math. 5 (1967), 249-271.

[N] K. Nowak, Ph.D. thesis, Washington Univ. in St. Louis, MO, 1991.

[R1] R. Rochberg, Toeplitz and Hankel operators, wavelets, NWO sequences, and almost diagonalization of operators (W. B. Arveson and R. G. Douglas, eds.), Proc. Sympos. Pure Math., vol. 51, Amer. Math. Soc., Providence, RI, 1990, pp. 425-444.

[R2] _ Eigenvalue estimates for Calderón-Toeplitz operators, preprint, 1990.

[Si] B. Simon, Trace ideals and their applications, Cambridge Univ. Press, London and New York, 1979.

[Z] K. Zhu, Operator theory in function spaces, Marcel Dekker, New York, 1990.

Department of Mathematics, Washington University in St. Louis, St. Louis, Missouri 63130

Current address: Institute of Mathematics, University of Wroclaw, pl. Grunwaldzki 2/4, 50-384 Wroclaw, Poland

E-mail address: knowak@plwruw11.bitnet 